\title{
Brief fixation does not hamper the reliability of Ki67 analysis in breast cancer core-needle biopsies: a double-centre study
}

Running title: Effect of brief fixation on Ki67 staining

Shona Kalkman ${ }^{1}$, Joris P. Bulte ${ }^{2}$, Altuna Halilovic ${ }^{3}$, Peter Bult ${ }^{3}$, Paul J. van Diest ${ }^{1}$

${ }^{1}$ Department of Pathology, University Medical Center Utrecht, Utrecht, The Netherlands.

${ }^{2}$ Department of Surgery, Radboud University Medical Center, Nijmegen, The Netherlands.

${ }^{3}$ Department of Pathology, Radboud University Medical Center, Nijmegen, The Netherlands.

Conflict of interest statement: The authors declare that they have no conflict of interest.

\section{Address for correspondence:}

Paul J. van Diest, MD, PhD

Professor and Head, Department of Pathology

University Medical Center Utrecht

PO Box 85500

3508 GA Utrecht

The Netherlands

Phone +31887556565

Fax $\quad+31887569593$

Email p.j.vandiest@umcutrecht.nl 


\section{Abstract}

Aims Immunohistochemical assessment of Ki67 expression in core-needle biopsies (CNBs) increasingly plays a role in therapeutic decision-making in breast cancer patients. Within the framework of same-day diagnostics of breast lesions, fixation times are markedly decreased. We therefore attempted to validate Ki67 analysis in briefly fixed breast cancer CNBs.

Methods and results CNBs of 136 consecutive patients with invasive breast cancer diagnosed through the same-day diagnostics programme of both the University Medical Center Utrecht (UMCU) and the Radboud University Medical Center (RUMC) were included. CNBs were fixed in formaldehyde for approximately 45 minutes in the UMCU and between 60 and 90 minutes in the RUMC. Immunohistochemistry for Ki67 expression was compared between the briefly fixed CNBS and conventionally fixed resection specimens of the same tumor. Overall agreement between CNBs and resections was 122/142 (85.9\%) for Ki67 expression ( $\kappa$ $=0.71 ; 95 \% \mathrm{CI}=0.60-0.83)$. Positive and negative predictive values were $79.7 \%(95 \% \mathrm{CI}=$ $0.67-0.88 \%)$ and $91.0 \%(95 \% \mathrm{CI}=0.82-0.96 \%)$, respectively.

Conclusions Overall agreement for Ki67 expression was good between briefly fixed CNBs and conventionally fixed resection specimens, and within the range of studies comparing conventionally fixed CNBs and resections.

Key words: fixation time, Ki67, cell proliferation, immunohistochemistry, core-needle biopsy, breast cancer 


\section{Introduction}

In personalized breast cancer care, various immunohistochemical analyses are nowadays applied for therapeutic decision-making. Hereto, markers reflecting proliferation are of great interest as they reflect risk of recurrence [1]. One of these markers associated with cell proliferation is the nuclear protein Ki67 which has strong prognostic value in breast cancer [2, 3]. Recently, the International Ki67 in Breast Cancer Working Group stressed the value of Ki67 as a predictor of prognosis and as a biomarker for treatment effectiveness [4]. Although not yet recommended in national guidelines, Ki67 appears to be widely determined in breast cancer tissue during routine clinical practice [5].

Demand is growing for the standardization of Ki67 assessment because of its important role in therapeutic decision-making. Immunohistochemistry has been widely adopted by different laboratories as the preferred detection method for Ki67. Nevertheless, preanalytical variables may still influence test results. In accordance with guidelines for hormone receptor assessment, a minimum fixation time of 6 hours in $10 \%$ neutral buffered formalin (NBF; is 4\% neutral buffered formaldehyde) is generally recommended for Ki67 [6]. Within the context of increasing demands for same-day diagnostics in breast cancer, speeding up the process of tissue handling for biomarker assessment is mandatory. There is already some evidence that brief fixation time does not influence Ki67 assessment in other tissue types. Munakata et al. studied fixation in NBF for 4 to 48 hours in one tonsil specimen and showed that fixation for only 4 hours was adequate for Ki67 analysis [7]. The aim of this doublecentre study was to assess whether even shorter fixation times (45-90 minutes) of same-day diagnostic CNBs allows for reliable immunohistochemical assessment of Ki67 expression in two cohorts of invasive breast cancer patients. Reporting was done according to the REMARK criteria [8]. 


\section{Materials and methods}

\section{Patients}

Since November 2011 and January 2008, the University Medical Center Utrecht (UMCU) and the Radboud University Medical Center (RUMC) [9], respectively, have been operating a same-day diagnosis programme for breast lesions. In this programme, patients with suspected malignancy are referred for analysis including CNB with the aim of informing patients about their diagnosis and suggested treatment regimen within one day. Cohorts of consecutively admitted patients with invasive breast cancer (with both CNB and subsequent resection performed at the same center) were included from both centres. As we used coded leftover material no ethical approval was required based on current Dutch legislation $[10,11]$. Patients undergoing neo-adjuvant therapy and with multifocal disease (the latter potentially providing sampling issues) were excluded from the study, unless correct matching of lesions on CNB and the resection could be thoroughly ensured. Mitotic activity index and tumor grade of resections was recovered from the databases in both centers to assess inter-laboratory differences between the cohorts.

\section{Tissue processing}

In the UMCU, CNBs were immediately placed in $\mathrm{NBF}$ and sent to the pathology laboratory to be registered (estimated prior fixation time 15 minutes). After registration, specimens were immediately placed in an automated tissue processor (Peloris ${ }^{\mathrm{TM}}$, Leica, Valkenswaard, The Netherlands), which started with a 30 minutes formaldehyde fixation step under vacuum (see Supporting information for complete protocol).

In the RUMC, CNBs were placed in Unifix $(3.7-4 \%$ formaldehyde solution containing zinc sulfate, acetate buffered at pH 5,6 - 5,8; Klinipath BV, Duiven, The Netherlands) and sent to the pathology laboratory to be registered (estimated prior fixation time 30 minutes). If this 
was less than 30 minutes, an extra fixation step of 30 minutes with formaldehyde was included. After registration, biopsy specimens were placed in a 'rapid microwave histoprocessor' (Pathos Delta, Milestone Srl, Sorisole, Italy), which included a 20 minutes formaldehyde fixation step ( 15 minutes up to $50^{\circ} \mathrm{C}$ and 5 minutes at a constant temperature of $50^{\circ} \mathrm{C}$ ) (see Supporting information for complete protocol).

Hereafter, in both centers tissue samples were routinely embedded in paraffin. Assuming a preprocessing formaldehyde exposure window (including transportation to the pathology laboratory and administration) of 15 minutes in the UMCU and 30-60 minutes in the RUMC, total formaldehyde fixation time before and during processing thereby varied between biopsies from 45 minutes to 90 minutes. Excision specimens were fixed overnight in compliance with the current ASCO/CAP guideline recommendations ( $\geq 6$ hours) [6]. Cold ischemia time in all included surgical specimens was less than two hours.

\section{Immunohistochemistry}

From the paraffin blocks of the tumors excised in the UMCU, one tissue microarray (TMA) was constructed by transferring tissue cylinders of $0.6 \mathrm{~mm}$ ( 3 cylinders per tumor) from the tumor area, determined by a pathologist based on haematoxylin/eosin stained slides, using a tissue arrayer (Beecher Instruments, Sun Prairie, WI, USA). Paraffin blocks of all biopsies and the TMA were cut into sections of $4 \mu \mathrm{m}$ and mounted (Table 1). In the UMCU, slides were baked at $37^{\circ} \mathrm{C}$ and manually incubated with mouse monoclonal antibodies against Ki67 (MIB-1, dilution 1:200) from DAKO (Glostrup, Denmark) at room temperature for 60 minutes (See Supporting information for complete staining protocol).

In the RUMC, resection specimens were cut into whole sections of $4 \mu \mathrm{m}$ thick and mounted.

Slides were baked at $56^{\circ} \mathrm{C}$ and then immunohistochemically stained by means of the Immunologic Autostainer 480 (Duiven, The Netherlands) using mouse monoclonal antibodies 
against Ki67 (MIB-1, dilution 1:100) (See Supporting information for complete staining protocol). Appropriate controls were used throughout.

\section{Scoring}

Ki67 percentage score was defined as the mean percentage of positively stained tumor nuclei (at any intensity) across all tumor cells of one slide of the same tumor at 100x magnification and subsequently at 40x magnification $[4,12]$. In each centre, percentage of positive nuclei was estimated by consensus scoring of two blinded observers including an experienced breast pathologist (UMCU: P.v.D. and S.K.; RUMC: P.B. and A.H.). Scoring was performed according to the following categories: $0 \%, 1 \%, 2 \%, 5 \%, 10 \%, 20 \%, 35 \%, 50 \%, 65 \%, 75 \%$, $90 \%$ and $100 \%$. A cut-off value of $15 \%$ for positive marker status was used according to current national and international recommendations $[2,3,13,14]$.

\section{Statistical analysis}

Overall agreement between the CNBs and resection specimens was calculated for Ki67 positive and negative values. Kappa statistics were calculated to estimate the level of agreement beyond chance. Values of $\kappa>0.6$, between 0.4 and 0.6 , between 0.2 and 0.4 , and $<$ 0.2 indicated good, moderate, fair and poor agreement, respectively [15]. Correlation between Ki67 values in CNB and resection specimens was calculated by means of Spearman's correlation coefficient, using quartiles $(<10 \%, 10-14 \%, 15-24 \%,>25 \%)$. To assess the accuracy of immunohistochemical tests on briefly fixed specimens in predicting either low or high proliferative fraction, we calculated positive and negative predictive values. In addition, mitotic activity scores and tumor grade distribution among resections of both cohorts were compared using Mann-Whitney tests. This was done to detect for differences between the two 
cohorts. All statistics were performed using IBM SPSS Statistics for Windows (version 20.0, IBM Corp., Armonk, NY, USA). 


\section{Results}

\section{UMCU results}

Between November 2011 and December 2012, 113 invasive breast cancers were diagnosed in the same-day CNB programme of the UMCU. A total of 72/113 (63.7\%) cases were eligible for analyses. Reasons for exclusion were the administration of neo-adjuvant therapy prior to surgery $(n=24)$, multifocal disease $(n=13)$ and the absence of remaining diagnostic material $(n=4)$.

Mean age of the patients was 60.6 years (ranging from 34 to 89 years). Mean time between CNB and surgery was 27.6 days (ranging from 7 to 51 days). A total of 43/72 (59.7\%) had invasive ductal carcinoma, 19/72 (26.4\%) invasive lobular carcinoma, 9/72 (12.5\%) invasive ductulolobular carcinoma and 1/72 (1.4\%) tubular carcinoma. In 6 patients, due to size, the CNB was divided over two cassettes allowing for analysis of both samples of the same CNB. Scores of the different slides of the same CNB were considered independent cases and were matched with the scores of the single corresponding resection specimens. Hence, $78 \mathrm{CNBs}$ and 72 corresponding surgical specimens were immunohistochemically stained for Ki67.

At immunohistochemical analysis, no deleterious effects due to processing were observed on the morphological preservation of the CNBs and staining intensity overall appeared no differently between CNBs and resection specimens. Five pairs were excluded that had no remaining tumor cells in the CNB or resection blocks. Thus, complete data of 68 patients (73 CNBs and 68 resections) were apt for further analysis. Ki67 expression was high in 7/68 $(10.3 \%)$ resections and in 17/73 (23.3\%) CNBs. Mean percentage of Ki67 positive tumor cells was $12 \%$ in the CNB group and $6 \%$ in the resection group. Overall agreement between CNB and resection was 64/73 (87.7\%) for Ki67 expression $(\kappa=0.58 ; 95 \% \mathrm{CI}=0.32-0.84)($ Table 2). There was a high correlation between Ki67 values in CNBs and corresponding resections $\left(r_{\mathrm{s}}=0.72 ; P<0.01\right)$. The positive and negative predictive values for CNB Ki67 expression 
were $47.1 \%(95 \% \mathrm{CI}=0.24-0.71)$ and $100.0 \%(95 \% \mathrm{CI}=0.92-1.00)$, respectively. Moderate correlation was observed between Ki67 values and mitotic activity index $\left(r_{\mathrm{s}}=0.53\right.$; $P<0.01)$ and between Ki67 values and tumor grade $\left(r_{\mathrm{s}}=0.57 ; P<0.01\right)$ in resections (Figure 1).

\section{RUMC results}

Between September 2012 and July 2013, 69 consecutive patients with 70 invasive breast cancers underwent CNB at the same-day diagnosis breast clinic of the RUMC. A total of 69 (98.6\%) cancers were eligible for analyses. One case $(1.4 \%)$ was excluded because no remaining tumor was present in the paraffin block of the CNB. Mean age of the included patients was 61.3 years (ranging from 35 to 87 years). A total of 57/69 (82.6\%) invasive cancers were invasive ductal carcinoma, 7/69 (10.1\%) invasive lobular carcinoma, 2/69 $(2.9 \%)$ tubular carcinoma, 2/69 (2.9\%) mucinous carcinoma, 1/69 (1.4\%) micropapillary invasive carcinoma and 1/69 (1.4\%) apocrine carcinoma. Sixty-nine CNBs and their corresponding resections were stained for Ki67.

At immunohistochemical analysis, morphology was considered unremarkable in the CNBs and no overall differences in staining intensity were observed between CNBs and resection specimens. Ki67 expression was high in 50/69 (72.5\%) resections and in 47/69 (68.1\%) CNBs. Mean percentage of Ki67 positive tumor cells was $26 \%$ in the CNB group and $25 \%$ in the resection group. Overall agreement between CNB and resection was 58/69 (84.1\%) for Ki67 expression $(\kappa=0.62 ; 95 \% \mathrm{CI}=0.41-0.83)$ (Table 2$)$. There was a high correlation between Ki67 values in CNBs and corresponding resections $\left(r_{\mathrm{s}}=0.76 ; P<0.01\right)$. The positive and negative predictive values for biopsy Ki67 expression were 91.5\% (95\% CI $=0.79-$ $0.97)$ and $68.2 \%(95 \% \mathrm{CI}=0.45-0.85)$, respectively. Moderate correlation was observed between Ki67 values and mitotic activity index $\left(r_{\mathrm{s}}=0.69 ; P<0.01\right)$ and between Ki67 values 
and tumor grade $\left(r_{\mathrm{s}}=0.61 ; P<0.01\right)$ in resections (Figure 2$)$. In the RUMC cohort, Ki67 values in resections were significantly higher than those in resections of the UMCU $(P<$ 0.01) (Table 3). Resections analysed in the RUMC were of significantly higher tumor grade than those analysed in the UMCU $(P<0.01)$. Mitotic activity scores were significantly higher in resections of the RUMC (median 11; IQR 4.00-21.00) compared with those in resections of the UMCU (median 5; IQR 2.25-14.00).

\section{Combined analysis of Ki67 comparability}

Overall agreement between pooled CNBs and resections was 122/142 (85.9\%) for Ki67 expression $(\kappa=0.71 ; 95 \% \mathrm{CI}=0.60-0.83)($ Table 2$)$. Positive and negative predictive values were $79.7 \%(95 \% \mathrm{CI}=0.67-0.88)$ and $91.0 \%(95 \% \mathrm{CI}=0.82-0.96)$, respectively. Correlation between Ki67 values in briefly fixed CNBs and conventionally fixed resections was high $\left(r_{\mathrm{s}}=0.82 ; P<0.01\right)$, when using quartiles to categorize data $(<10 \%, 10-14 \%, 15-$ $24 \%,>25 \%$ ). Table 4 shows the degree of variation between discordant cases as well as the predominant histological subtypes. 


\section{Discussion}

In this double-centre study, agreement between briefly fixed CNBs and conventionally fixed resections was good with an observed $\kappa$ value of $0.71(95 \% \mathrm{CI}=0.60-0.83)$ and a Spearman correlation coefficient of $0.82(P<0.01)$. When comparing the present findings with previous research on agreement levels between conventionally fixed CNBs and resections, at least a similar level of concordance was seen. Romero et al. found an overall agreement of $76 \%$ for a set of 50 paired samples. The authors noted that $20 \%$ of CNBs converted from positive to negative in the surgical specimens as opposed to a $4 \%$ conversion from negative to positive ( $P$ $=0.039)[16]$. A concordance rate of $73 \%$ with a $\kappa$ value of 0.48 was reported by Greer et al. [17]. Chen et al. found a concordance rate of $79.5 \%(\kappa=0.55)$ for CNBs and surgical samples $(n=298)$ [18]. Therefore, our findings indicate that brief fixation of breast cancer biopsies does not seem to hamper Ki67 assessment.

Regarding Ki67 scoring inconsistencies in this study as well as in general, a few other factors may have accounted for the observed differences. A first factor that may have contributed to Ki67 scoring inconsistency is observer variation. Vörös et al. showed that Ki67 values were significantly influenced by the observer even if standardised criteria were used during the scoring process. Not only the inter-observer, but also the intra-observer agreement was found to be poor to moderate. Intermediate categories were generally less reproducible than low and high value categories [19]. High agreement levels reported for estrogen receptor expression between conventionally fixed CNBs and resections [20] suggest that estimating percentages of generally homogenously distributed positively stained nuclei may in itself be well reproducible, and that the much more heterogeneous distribution of Ki67 compared to other nuclear biomarkers inherently hampers reproducibility. In the present study, observer variation was minimal since all paired specimens (CNB and resection) were scored by two observers: in the UMCU cohort by consensus, and in the RUMC cohort by two observers with 
excellent inter-observer agreement (weighted $\kappa=0.88 ;(95 \% \mathrm{CI}=0.84-0.93 \%))$. In all discrepant cases between the two observers in the RUMC consensus was reached after evaluation of the Ki67 stained slides together with a biheaded microscope.

Agreement between CNBs and resection specimens was slightly better in the RUMC cohort, in which two factors may have played a role. First, in the UMCU cohort, CNBs were compared with three $0.6 \mathrm{~mm}$ TMA cores of the resection of the same tumor. It is conceivable that a certain sampling error was generated because of this method, explaining perhaps the generally lower Ki67 values in the resection specimens in this cohort. There are no published systematic comparisons of the assessment of Ki67 on TMAs versus whole sections, but the International Ki67 in Breast Cancer Working Group speaks of anecdotal evidence that scores are generally lower on TMAs [4]. This may be viewed as a methodological disadvantage, as the TMA cores add up to a smaller tissue surface than the amount of material that an average CNB slide contains, potentially leading to an underestimation of Ki67 fraction. However, one may argue that the alternative (use of whole sections from the resections) could have had the opposite effect. Second, overall Ki67 values in resections were rather low in the UMCU cohort (which is a screening population with also lower grade and mitotic activity) where reproducibility is more difficult to achieve, while in the RUMC the Ki67 values (and grade and mitotic index) were higher with inherently higher reproducibility. The difference in agreement was however small $\left(\kappa=0.58\right.$ versus $\kappa=0.62, r_{\mathrm{s}}=0.72$ versus $\left.r_{\mathrm{s}}=0.76\right)$, indicating that other methodological variations (slight differences in tissue processing and staining protocols) must be minimal. Preferably, we would have performed a parallel comparison between Ki67 percentage scores in conventionally fixed CNBs and those scores in the corresponding conventionally fixed resection specimens. In doing so, we could have provided a match-to-match baseline for assessing the efficacy of estimating Ki67 status in briefly fixed CNBs. However, as the CNBs were taken in the context of clinical care performing extra 
CNBs that were to be fixed conventionally for research purposes would have put an extra burden on patients. Instead, we compared our findings with the agreement levels between conventionally fixed CNBs and conventionally fixed resections specimens that have been reported in the literature.

Persisting inconsistencies in Ki67 analysis for conventionally fixed CNBs and resections and observer variation within the same sample, imply that implementation of Ki67 analysis in routine practice is still fallible. Augmenting our understanding of the biological behaviour of Ki67 as an immunohistochemical biomarker will most likely contribute to future standardization. Hereto, it appears necessary to differentiate between preanalytical factors that may be modified without adversely affecting Ki67 assessment and which may not.

In conclusion, overall agreement for Ki67 expression was good between briefly fixed CNBs and conventionally fixed resection specimens, within the range of previous studies comparing conventionally fixed CNBs and resections. Therefore, it is unlikely that brief fixation as preanalytical variable accounts for observed differences in this study. Rather, tumor heterogeneity, sampling error and lower reproducibility of scoring in the low and intermediate categories appear to be responsible for scoring inconsistencies. Therefore, we conclude that brief fixation does not seem to hamper reliability of Ki67 analysis in breast cancer CNB.

Acknowledgements: All authors contributed extensively to the work presented in this paper. P.v.D conceived the study and jointly designed the experiments with S.K. Experiments were performed by A.H. and S.K. with assistance of Natalie ter Hoeve (UMCU), Monique Link and Lambert Burgers (RUMC). S.K., J.B., A.H., P.B. and P.v.D. performed data collection and analysis. S.K. and P.v.D. wrote the main manuscript. All authors discussed the results and implications and commented on the manuscript at all stages. 
Funding: None. 


\section{References}

[1] Van Diest PJ, Van der Wall E, Baak JPA. Prognostic value of proliferation in invasive breast cancer: a review. J Clin Pathol 2004; 57: 675-681.

[2] Yerushalmi R, Woods R, Ravdin PM, Hayes MM, Gelmon KA. Ki67 in breast cancer: prognostic and predictive potential. Lancet Oncol 2010; 11: 174-83.

[3] Stuart-Harris R, Caldas C, Pinder SE, Pharoah P. Proliferation markers and survival in early breast cancer: a systematic review and meta-analysis of 85 studies in 32,825 patients. Breast 2008; 17: 323-34.

[4] Dowsett M, Nielsen TO, A'Hern R et al; International Ki-67 in Breast Cancer Working Group . Assessment of Ki67 in breast cancer: recommendations from the International Ki67 in Breast Cancer Working Group. J Natl Cancer Inst 2011; 103: 1656-1664.

[5] Inwald EC, Klinkhammer-Schalke M, Hofstädter F et al. Ki-67 is a prognostic parameter in breast cancer patients: results of a large population-based cohort of a cancer registry. Breast Cancer Res Treat 2013; 139(2): 539-52.

[6] Hammond ME, Hayes DF, Dowsett $M$ et al. American Society of Clinical Oncology/College Of American Pathologists guideline recommendations for immunohistochemical testing of estrogen and progesterone receptors in breast cancer. J Clin Oncol 2010; 28(16): 2784-95.

[7] Munakata S, Hendricks JB. Effect of fixation time and microwave oven heating time on retrieval of the Ki-67 antigen from paraffin-embedded tissue. J Histochem Cytochem 1993; 41(8): 1241-1246.

[8] McShane LM, Altman DG, Sauerbrei W, Taube SE, Gion M, Clark GM; Statistics Subcommittee of NCI-EORTC Working Group on Cancer Diagnostics. REporting recommendations for tumor MARKer prognostic studies (REMARK). Breast Cancer Res Treat 2006; 100(2): 229-35. 
[9] Bulte JP, Polman L, Schlooz-Vries M et al. One-day core needle biopsy in a breast clinic: 4-years experience. Breast Cancer Res Treat 2013; 137: 609-616.

[10] The Medical Research Involving Human Subjects Act (Wet medisch-wetenschappelijk onderzoek met mensen, WMO Central Committee on Research involving Human Subjects (Centrale Commissie Mensgebonden Onderzoek) (text in Dutch). Available at: http://www.ccmo.nl/en/non-wmo-research. Accessed 2 April 2014

[11] Van Diest PJ. No consent should be needed for using leftover body material for scientific purposes. BMJ 2002; 325: 648-651.

[12] Urruticoechea A, Smith IE, Dowsett M. Proliferation marker Ki-67 in early breast cancer. J Clin Oncol 2005; 23: 7212-7220.

[13] Goldhirsch A, Wood WC, Coates AS et al. Strategies for subtypes-dealing with the diversity of breast cancer: highlights of the St. Gallen International Expert Consensus on the Primary Therapy of Early Breast Cancer 2011. Ann Oncol 2011; 22: 1736-1747.

[14] de Azambuja E, Cardoso F, de Castro G Jr et al. Ki-67 as prognostic marker in early breast cancer: a meta-analysis of published studies involving 12,155 patients. Br J Cancer 2007; 96(10): 1504-1513.

[15] Landis JR, Koch GG . The measurement of observer agreement for categorical data. Biometrics 1977; 33: 159-74.

[16] Romero Q, Bendahl PO, Klintman M et al. Ki67 proliferation in core biopsies versus surgical samples - a model for neo-adjuvant breast cancer studies. BMC Cancer 2011; 11: 341.

[17] Greer LT, Rosman M, Mylander WC et al. Does breast tumor heterogeneity necessitate further immunohistochemical staining on surgical specimens? J Am Coll Surg 2013; 216(2): $239-251$. 
[18] Chen X, Sun L, Mao Y et al. Preoperative core needle biopsy is accurate in determining molecular subtypes in invasive breast cancer. BMC Cancer 2013; 13: 390.

[19] Vörös A, Csörgő E, Nyári T, Cserni G. An intra- and interobserver reproducibility analysis of the Ki-67 proliferation marker assessment on core biopsies of breast cancer patients and its potential clinical implications. Pathobiology 2013; 80(3): 111-8.

[20] Li S, Yang X, Zhang Y et al. Assessment accuracy of core needle biopsy for hormone receptors in breast cancer: a meta-analysis. Breast Cancer Res Treat 2012; 135(2): 325-34. 
Table 1. Ki67 analysis methodology for comparing briefly fixed CNB and conventionally fixed resection specimens.

\begin{tabular}{l|l|l}
\hline Centre & \multicolumn{1}{|c|}{ UMCU } & \multicolumn{1}{c}{ RUMC } \\
\hline Patients $(n)$ & 72 & 69 \\
CNBs $(n)$ & 78 & 70 \\
Resections $(n)$ & 72 & 70 \\
Antibody & Mouse MoAb, clone MIB-1 & Mouse MoAb, clone MIB-1 \\
Proportion CNB & Whole sections & Whole sections \\
$\begin{array}{l}\text { Proportion } \\
\text { resection }\end{array}$ & Tissue \\
Staining protocol & microarray & Whole sections \\
Consensus scoring by & Manually & Autostainer \\
\hline
\end{tabular}


Table 2. Comparison of Ki67 expression analysis between briefly fixed CNB and conventionally fixed resection specimens in two breast cancer cohorts.

\begin{tabular}{|c|c|c|c|}
\hline Centre & UMCU & RUMC & Combined \\
\hline$\%$ Positive in CNB $(n)$ & $23.3(17 / 73)$ & $68.1(47 / 69)$ & $45.1(64 / 142)$ \\
\hline Mean \% CNBs & 12 & 26 & 21 \\
\hline$\%$ Positive in resection $(n)$ & $10.3(7 / 68)$ & $72.5(50 / 69)$ & $41.6(57 / 137)$ \\
\hline Mean $\%$ resections & 6 & 25 & 15 \\
\hline Overall agreement (\%) & $87.7(64 / 73)$ & $84.1(58 / 69)$ & $85.9(122 / 142)$ \\
\hline Kappa $(95 \%$ CI $)$ & $0.58(0.32-0.84)$ & $0.62(0.41-0.83)$ & $0.71(0.60-0.83)$ \\
\hline$\%$ PPV $(95 \%$ CI $)$ & $47.1(0.24-0.71)$ & $91.5(0.79-0.97)$ & $79.7(0.67-0.88)$ \\
\hline$\%$ NPV $(95 \% \mathrm{CI})$ & $100.0(0.92-1.00)$ & $68.2(0.45-0.85)$ & $91.0(0.82-0.96)$ \\
\hline $\begin{array}{l}\text { Spearman's } \rho \text { CNBs and } \\
\text { resections }\end{array}$ & $0.72(P<0.01)$ & $0.76(P<0.01)$ & $0.82(P<0.01)$ \\
\hline
\end{tabular}

Abbreviations: $C N B$, core-needle biopsy; $P P V$, positive predictive value; $N P V$, negative predictive value; $95 \%$ CI, 95\% confidence interval; Spearman's $\rho$, correlation coefficient; $P, P$ value for significance.

Table 3. Comparison of tumor grade distribution, mitotic activity and Ki67 values of resections between the two cohorts.

\begin{tabular}{l|c|c|c|c}
\hline Centre & & UMCU (n= 68) & RUMC (n= 69) & P-value \\
\hline Grade & I & $22(32.4 \%)$ & $13(18.8 \%)$ & \\
& II & $30(44.1 \%)$ & $25(36.2 \%)$ & $<0.01$ \\
& III & $16(23.5 \%)$ & $31(44.9 \%)$ & \\
Median Ki67 expression (\%) & & $3.5(\mathrm{IQR} 2.00-8.75)$ & $20(\mathrm{IQR} 10.00-35.00)$ & $<0.01$ \\
Median mitotic activity $\left(n / 2 \mathrm{~mm}^{2}\right)$ & & $5(\mathrm{IQR} 2.25-14.00)$ & $11(\mathrm{IQR} 4.00-21.00)$ & $<0.01$ \\
\hline
\end{tabular}

Abbreviations: $I Q R$, interquartile range; $n$, number; $P, P$ value for significance. 
Table 4. Ki67 discordant cases $(n=20)$.

\begin{tabular}{c|c|c|c|c|c}
\hline Degree of variation & $\boldsymbol{n}$ cases & $\mathbf{C N B}>\mathbf{R}$ & IDC & ILC & Other \\
\hline$\leq 10 \%$ & $13 / 20(65,0 \%)$ & $8 / 13(61,5 \%)$ & $9 / 13(69,2 \%)$ & $3 / 13(23.1 \%)$ & $1 / 13(7,7 \%)$ \\
$>10-30 \%$ & $6 / 20(30,0 \%)$ & $4 / 6(66,7 \%)$ & $5 / 6(83,3 \%)$ & $1 / 6(16.7 \%)$ & - \\
$>30 \%$ & $1 / 20(5,0 \%)$ & $1 / 1(100 \%)$ & $1 / 1(100 \%)$ & - & - \\
Total & $20(100 \%)$ & $13 / 20(65,0 \%)$ & $15 / 20(75,0 \%)$ & $4 / 20(20,0 \%)$ & $1 / 20(5,0 \%)$ \\
\hline
\end{tabular}

Abbreviations: $n$, number; $C N B>R$, positivity score higher in core-needle biopsy than in resection specimen; IDC, invasive ductal carcinoma; $I L C$, invasive lobular carcinoma; Other, other histological subtype. 
Table 4.

\begin{tabular}{|c|c|c|c|}
\hline Ki67 expression CNB & Ki67 expression resection & Degree of variation & Histological subtype \\
\hline 35 & 5 & 30 & IDC \\
\hline 20 & 10 & 10 & IDC \\
\hline 20 & 5 & 15 & IDC \\
\hline 35 & 10 & 25 & IDC \\
\hline 90 & 10 & 80 & IDC \\
\hline 20 & 10 & 10 & IDC \\
\hline 20 & 10 & 10 & IDC \\
\hline 20 & 10 & 10 & IDC \\
\hline 20 & 10 & 10 & ILC \\
\hline 2 & 20 & 18 & IDC \\
\hline 10 & 20 & 10 & ILC \\
\hline 20 & 5 & 15 & ILC \\
\hline 10 & 20 & 10 & IDC \\
\hline 10 & 20 & 10 & IDC \\
\hline 10 & 20 & 10 & IDC \\
\hline 20 & 10 & 10 & IDC \\
\hline 20 & 10 & 10 & IDC \\
\hline 20 & 10 & 10 & Mucinous \\
\hline 10 & 35 & 25 & IDC \\
\hline 10 & 20 & 10 & ILC \\
\hline
\end{tabular}




\section{Figure Legends}

Figure 1: Two Ki67 discordant cases of the UMCU with high $(\geq 15 \%)$ Ki67 expression values in briefly fixed biopsies (top and bottom left) but low expression values in corresponding resection specimens (top and bottom right); (all parts $\times 200)$.

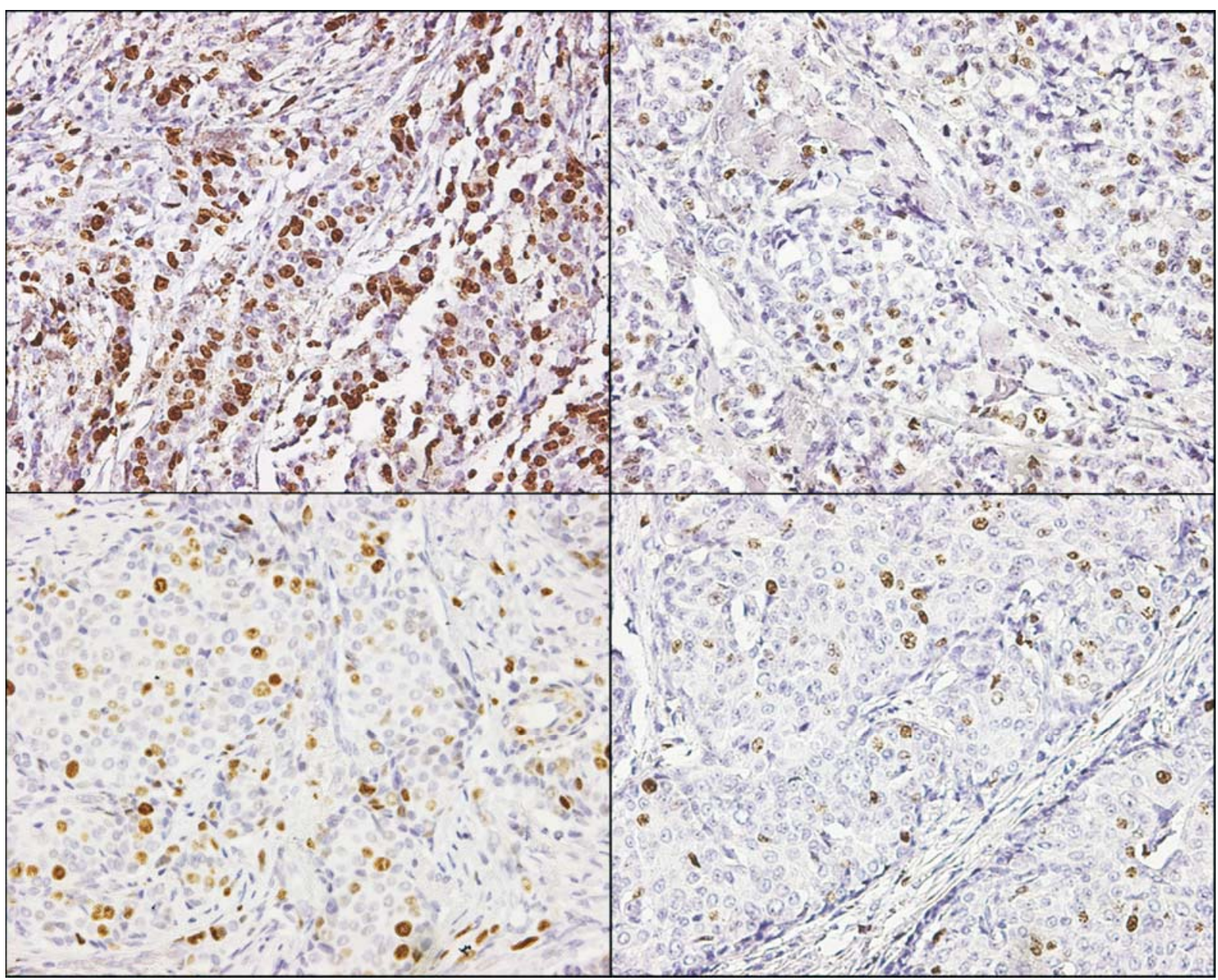


Figure 2: One Ki67 discordant and one Ki67 concordant case of the RUMC. Low $(<15 \%)$

Ki67 expression values in a briefly fixed biopsy (top left) but high expression values in the corresponding resection specimen (top right); low Ki67 expression values in a briefly fixed biopsy (bottom left) and low expression values in the corresponding resection specimen (bottom right); (all parts $\times 200)$.

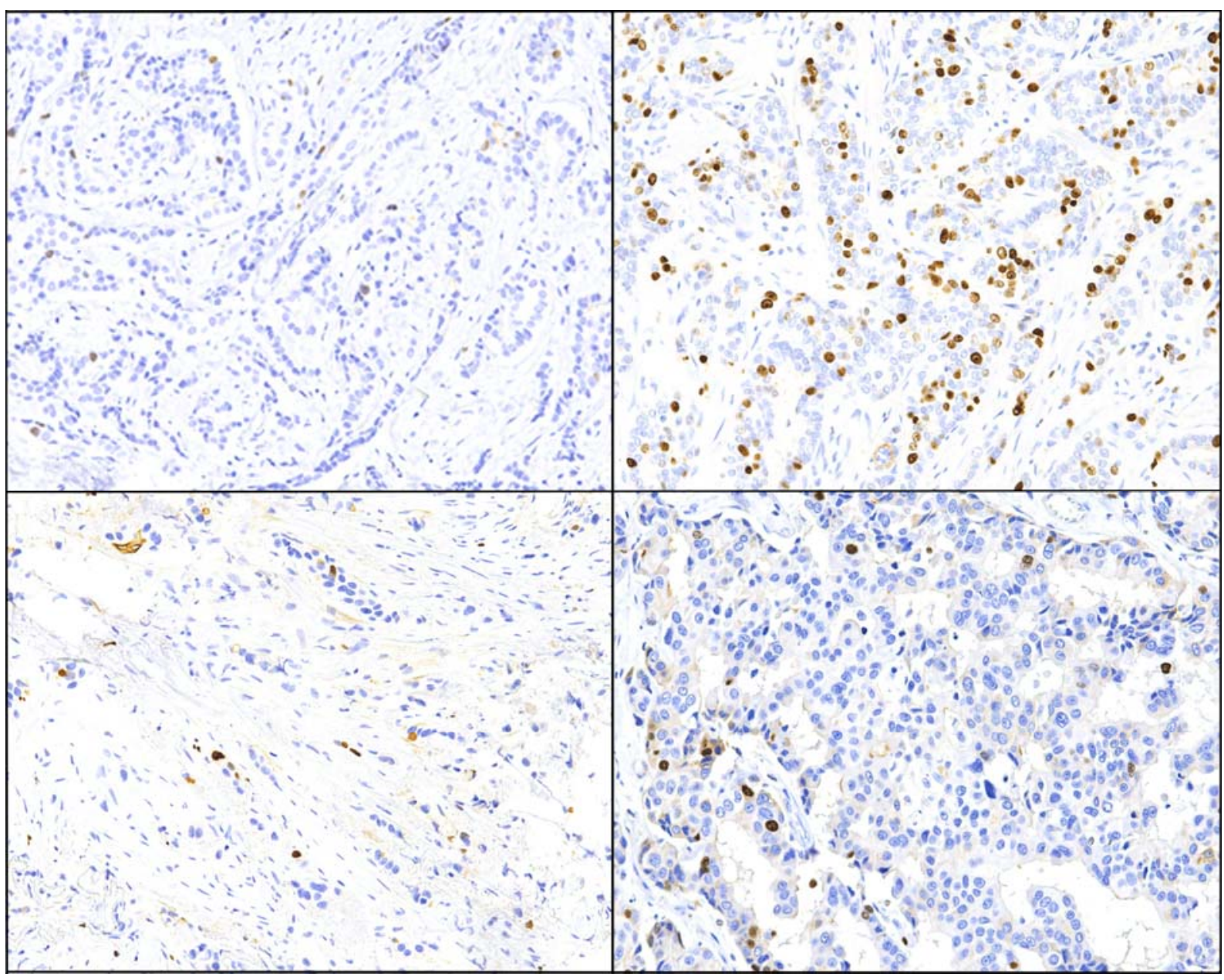

